SHORT REPORT

\title{
Chemokine response in children with SARS
}

\section{P C Ng, C W K Lam, A M Li, C K Wong, T F Leung, F W T Cheng, K L E Hon, I H S Chan, E Wong, T F Fok}

The chemokine response of eight children with serologically confirmed severe acute respiratory syndrome (SARS) was longitudinally monitored. All had raised plasma interferon $\gamma$ inducible protein (IP-10) concentrations, which suggested an active type 1 T-helper lymphocyte mediated immune response. High circulating IP-10 levels could facilitate viral clearance and might play a role in assisting the recovery of the patients.

C hemokines are secreted proteins that regulate the trafficking of specific populations of leucocytes to the site of injury and infection. Growing evidence suggests that these proinflammatory molecules represent an important component in the host defence by initiating specific immunological reactions. We have longitudinally monitored a panel of key chemokines, including interferon $\gamma$ inducible protein 10 (IP-10), monokine induced by interferon $\gamma$ (MIG), monocyte chemoattractant protein 1 (MCP-1), and regulated upon activation normal $\mathrm{T}$ cell expressed and secreted (RANTES) in a cohort of children with severe acute respiratory syndrome (SARS). The evaluation of inflammatory response to SARS coronavirus (SARS-CoV) is crucial for understanding the mechanisms of immune protection.

\section{PATIENTS AND METHODS}

The clinical, radiological, laboratory, and microbiological features of eight children with serologically confirmed SARS were described in detail in our previous report; the case numbers assigned to these children also corresponded with those in the same study. ${ }^{1}$ In brief, case 3 was the only patient who did not receive corticosteroid treatment. Cases 2 and 6 required pulse methylprednisolone, and case 6 also received intravenous ribavirin. All patients showed clinical improvement with subsidence of fever and complete or partial resolution of chest radiological changes, 2-7 days after corticosteroid treatment. None required assisted ventilation and all patients survived.

Blood samples for chemokine measurement were obtained on admission, immediately (within 24 hours) before starting corticosteroids, and 1-2 days, 7-10 days, and 3-6 weeks after drug treatment. A panel of chemokines, including IP-10, MIG, MCP-1, and RANTES, were simultaneously quantified by the Human Chemokine Cytometric Bead Array Kit (BD Pharmingen, San Diego, CA) using flow cytometry and following the manufacturer's protocol. Their assay sensitivities were $2.8,2.5,2.7$, and $1.0 \mathrm{ng} / \mathrm{l}$ respectively, and the coefficients of variation for all assays were less than $10 \%$.

The Wilcoxon signed rank test was used to assess the difference in plasma chemokine concentrations immediately before and at different time points after commencement of corticosteroids. The study was approved by the Research Ethics Committee of the Chinese University of Hong Kong.

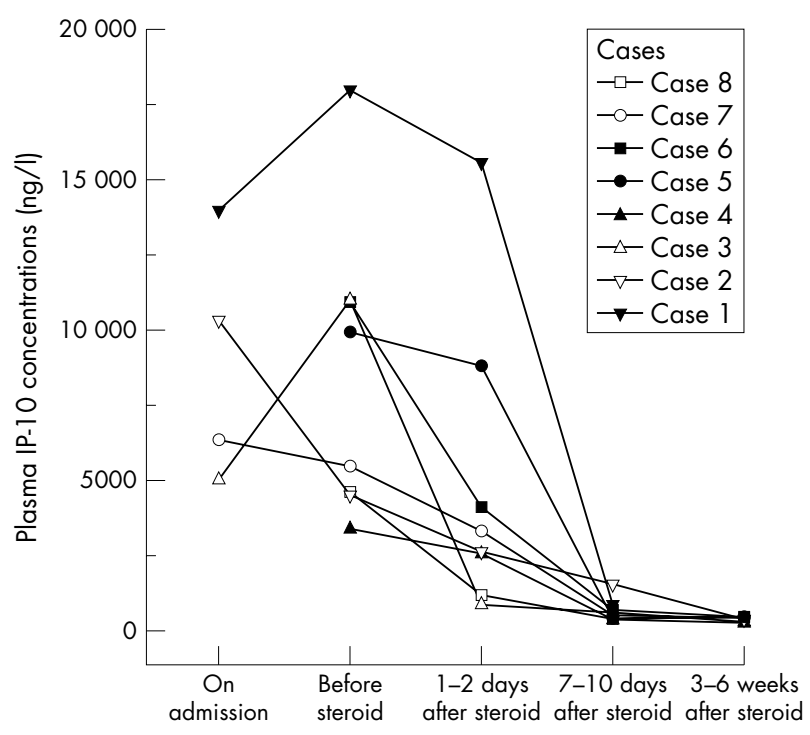

Figure 1 The change in plasma IP-10 concentrations at five different time points after the onset of illness-on admission, immediately before corticosteroid treatment, and 1-2 days, 7-10 days, and 3-6 weeks after treatment-in eight children with SARS-CoV infection. All patients except case 3 received corticosteroids. The blood samples were obtained from case 3 for plasma chemokine measurements on days $3,8,10,14$, and 29 after the onset of fever.

Informed consent was obtained from the parents of all patients.

\section{RESULTS}

Four children (cases 4, 5, 6, and 8) did not have blood taken for chemokines on admission, and the youngest child (case 1) did not receive the final blood test. Table 1 summarises the chemokine results. Plasma IP-10 concentrations were substantially raised in all corticosteroid treated patients immediately before (range 3434-18 014 ng/l) and 1-2 days after the drug treatment (range 1247-15 $591 \mathrm{ng} / \mathrm{l}$; see fig l). Plasma levels of MIG (range 149-988 ng/l) and MCP-1 (range 18-578 ng/l) were only mildly increased in the acute phase of illness. Five and two of the seven corticosteroid treated patients had abnormally increased plasma MIG and MCP-1, respectively. The overall trend suggested increased levels at the initial phase which were then followed by a decline in plasma concentrations with time. Plasma RANTES levels varied widely (range 4852-37 $220 \mathrm{ng} / \mathrm{l}$ ), but did not increase above the normal reference range in any of the patients.

Abbreviations: IP-10, interferon $\gamma$ inducible protein; MCP-1, monocyte chemoattractant protein 1; MIG, monokine induced by interferon $\gamma$; RANTES, regulated on activation normal T cell expressed and secreted; SARS, severe acute respiratory syndrome; SARS-CoV, SARS coronavirus; Th1, type $1 \mathrm{~T}$ helper 
Table 1 Changes in chemokine profile of children with SARS (excluding case 3) before and after corticosteroid treatment

\begin{tabular}{|c|c|c|c|c|c|}
\hline $\begin{array}{l}\text { Plasma chemokine } \\
\text { concentrations (ng/l) }\end{array}$ & $\begin{array}{l}\text { Immediately before } \\
\text { corticosteroid treatment } \\
(\mathrm{n}=7)\end{array}$ & $\begin{array}{l}1-2 \text { days after } \\
\text { corticosteroid treatment } \\
(n=7)\end{array}$ & $\begin{array}{l}\text { 7-10 days after } \\
\text { corticosteroid treatment } \\
(n=7)\end{array}$ & $\begin{array}{l}\text { 3-6 weeks after } \\
\text { corticosteroid treatment } \\
(\mathrm{n}=6)\end{array}$ & $\begin{array}{l}\text { Normal reference } \\
\text { range }\end{array}$ \\
\hline IP-10 & 5503 (4506-10877) & $3373(2621-8889)^{*}$ & $660(438-929)^{*}$ & $489(379-535)^{*}$ & $232-1019$ \\
\hline MIG & 421 (397-878) & 509 (405-878) & $486(174-575)$ & 200 (170-293)* & $37-463$ \\
\hline MCP-1 & $108(84-182)$ & $93(47-134)$ & $47(33-66)^{*}$ & $53(23-78)^{*}$ & $18-152$ \\
\hline RANTES & 26979 (21160-29192) & $25613(11661-35233)$ & $33369(22252-36711)$ & $22974(16686-35020)$ & $10349-46704$ \\
\hline
\end{tabular}

Results are median (interquartile range).

${ }^{*} \mathrm{p}<0.05$ (comparison of plasma chemokine concentrations before and after corticosteroid treatment).

\section{DISCUSSION}

The most striking phenomenon was the increase in plasma IP-10, and to a lesser extent, MIG concentrations during the acute phase of the disease which was followed by normalisation of levels in the convalescent period (fig 1 and table 1). As IP-10 and MIG are potent chemoattractants for activated type $1 \mathrm{~T}$ helper (Thl) lymphocytes, our findings suggest that SARS-CoV activates mainly the Thl immune response against viral invasion. Early and prominent expression of IP-10 and MIG have been shown to be beneficial to the host in a rodent model infected with neurotropic coronavirus by: (1) attracting activated Thl lymphocytes to the site of infection; (2) maintaining a high level of interferon $\gamma$ at the site, thereby facilitating viral clearance; and (3) increasing the survival in infected animals. ${ }^{2}$ In contrast, treatment of infected mice with anti-IP-10 antiserum or anti-MIG antiserum resulted in impaired host $\mathrm{T}$ cell response and increased mortality. ${ }^{2}$

The chemokine profile of SARS-CoV is different from those of influenza A virus, ${ }^{3}$ Sendai virus, ${ }^{3}$ and respiratory syncytial virus $^{4}$ in that RANTES production was not up-regulated. As RANTES is a potent eosinophil and memory T cell chemoattractant and activator, it plays an important role in the development of recurrent bronchospasm after an acute respiratory viral infection. ${ }^{4}$ The absence of a significant upsurge in plasma RANTES in children with SARS correlates closely with the clinical observations that (1) none of the children in our cohort has recurrent wheezy attacks after the infection, and (2) only two of 38 asymptomatic children who underwent pulmonary function assessment six months after the acute infection showed mild obstructive pattern of impairment. Thus, the immunological profile probably suggested less involvement of the Th2 lymphocyte mediated response in SARS-CoV infection.

There are limitations in this study. Firstly, the plasma chemokine levels were only monitored in a small number of paediatric patients; however, their disease pattern was a good reflection of paediatric SARS, as the great majority (96\%) of affected children did not require intensive care treatment in Hong Kong. Further, this pattern of chemokine response is similar to that observed in adult SARS patients. ${ }^{5}$ Secondly, in the absence of a matched control group, it is difficult to be certain whether the decline in chemokine levels with time was associated with corticosteroid treatment or simply following the natural recovery process of the disease. It is, however, worth noting that circulating chemokines in case 3 (the only patient who did not receive corticosteroids) also followed a similar decreasing trend (fig 1).

In summary, the substantial increase in plasma IP-10 suggests an active Thl mediated antiviral response to SARSCoV. High circulating IP-10 can facilitate viral clearance and may play a role in assisting the recovery of the patients. The lack of a significant upsurge in circulating RANTES level is unexpected, but correlates with our clinical observation that the majority of patients do not have clinical manifestations or spirometry features suggestive of postviral obstructive airway disease.

\section{Authors' affiliations}

P C Ng, A M Li, T F Leung, F W T Cheng, K L E Hon, T F Fok, Department of Paediatrics, Prince of Wales Hospital, The Chinese University of Hong Kong

C W K Lam, C K Wong, I H S Chan, Department of Chemical Pathology, Prince of Wales Hospital, The Chinese University of Hong Kong E Wong, Centre for Clinical Trials and Epidemiological Research, Prince of Wales Hospital, The Chinese University of Hong Kong

Funding: This project is funded by the Department of Chemical Pathology, The Chinese University of Hong Kong

Competing interests: none declared

Correspondence to: Prof. P C Ng, Department of Paediatrics, Level 6, Clinical Sciences Building, Prince of Wales Hospital, Shatin, New Territories, Hong Kong; pakcheungng@cuhk.edu.hk

Accepted 11 August 2004

\section{REFERENCES}

$1 \mathrm{Ng}$ PC, Lam CWK, Li AM, et al. Inflammatory cytokine profile in children with severe acute respiratory syndrome. Pediatrics 2004;113:e7-14.

2 Liu MT, Chen BP, Oertel P, et al. The T cell chemoattractant IFN-inducible protein 10 is essential in host defense against viral-induced neurologic disease. J Immunol 2000;165:2327-30.

3 Matikainen S, Pirhonen J, Miettinen $M$, et al. Influenza $A$ and Sendai viruses induce differential chemokine gene expression and transcription factor activation in human macrophages. Virology 2000;276:138-47.

4 Chung HL, Kim SG. RANTES may be predictive of later recurrent wheezing after respiratory syncytial virus bronchiolitis in infants. Ann Allergy Asthma Immunol 2002;88:463-7.

5 Wong CK, Lam CWK, Wu AK, et al. Plasma inflammatory cytokines and chemokines in severe acute respiratory syndrome. Clin Exp Immunol 2004;136:95-103. 